\title{
A Design Framework for Playful Wearables
}

\author{
Oğuz 'Oz’ Buruk \\ Tampere University \\ Tampere, Finland \\ oguz.buruk@tuni.fi
}

\author{
Katherine Isbister \\ University of California, Santa Cruz \\ Santa Cruz, CA, USA \\ katherine.isbister@ucsc.edu
}

\author{
Theresa Jean Tanenbaum \\ University of California, Irvine \\ Irvine, $\mathrm{CA}$, USA \\ ttanen@uci.edu
}

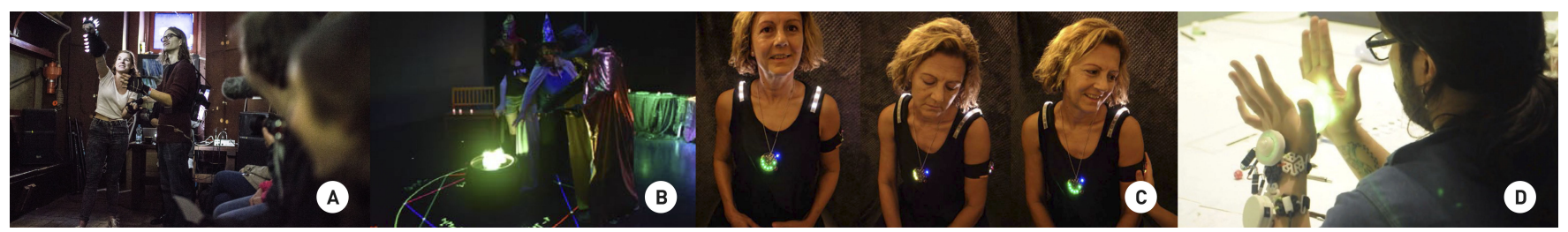

Figure 1: A) Two players are trying Hotaru (Photo by Martin Christopher Welker for Playpublik Festival Krakow 2014). B) Spell casting scene from Magia Transformo. C) Mental Energy and Health Modules of SW4LARP. D) Concentration Game in WEARPG

\begin{abstract}
Deployment of wearables for games has attracted the interest of designers and researchers both in academia and industry. However, few of these projects treat wearables as an integral part of the gameplay, often considering them as an extension of the central on-screen experience. While preliminary forays into wearable play show promise, we see a need for a rigorous design framework to illuminate the possibilities for the future of wearables for playful interaction design. In this paper we propose a Design Framework for Playful Wearables stemming from our extensive research and hands-on experience in leading four long-term game research projects incorporating wearables. We divide our framework into three high-level categories: the performative, the social and the interactive. We contend that this design space can be both a design tool for creators of wearable playful activities, as well as an analytical lens for evaluating existing wearable systems.
\end{abstract}

\section{CCS CONCEPTS}

- Human-centered computing $\rightarrow$ User interface design; Interface design prototyping; User centered design;

\section{KEYWORDS}

Wearables, Playful Interaction, Game Research, Movement-Based Games, Costuming, Role Playing, RPG, LARP

\section{ACM Reference Format:}

Oğuz ‘Oz’ Buruk, Katherine Isbister, and Theresa Jean Tanenbaum. 2019. A Design Framework for Playful Wearables. In The Fourteenth International Conference on the Foundations of Digital Games (FDG '19), August 26-30, 2019, San Luis Obispo, CA, USA. ACM, New York, NY, USA, 12 pages. https://doi.org/10.1145/3337722.3337733

Permission to make digital or hard copies of all or part of this work for personal or classroom use is granted without fee provided that copies are not made or distributed for profit or commercial advantage and that copies bear this notice and the full citation on the first page. Copyrights for components of this work owned by others than the author(s) must be honored. Abstracting with credit is permitted. To copy otherwise, or republish, to post on servers or to redistribute to lists, requires prior specific permission and/or a fee. Request permissions from permissions@acm.org.

FDG '19, August 26-30, 2019, San Luis Obispo, CA, USA

(c) 2019 Copyright held by the owner/author(s). Publication rights licensed to ACM.

ACM ISBN 978-1-4503-7217-6/19/08 ..\$15.00

https://doi.org/10.1145/3337722.3337733

\section{INTRODUCTION}

Embodied gameplay has become increasingly common as computation, sensors, and network capacity have made building and deploying such systems more tenable. The inclusion of movement-based gameplay hardware in many of the major consoles over the last decade (such as the Kinect, PS Move, and Wii) was a precursor to the emerging embodied interactions and controllers of the current generation of virtual reality (VR) systems. The HTC Vive, and Oculus Rift, and Windows MR, all use motion tracking technology for full body gameplay. Gaming's history is also rich with examples of embodied play using specialized and "one-off" controllers, such as Dance Dance Revolution, and rhythm and music games like Rock Band and Guitar Hero [49].

Wearables can be seen as an extension of this trend. Incorporating wearable devices into play provides unique opportunities such as continuous and independent body tracking, the ability to track bodyrelated data such as motion and biometrics, the addition of a tangible layer to the game, and the fostering of immersion and connection. The research community has been creating and studying wearables for some time $[2,36,53]$. And more recently, wearables have begun to make major commercial appearances. Examples include the wrist-worn Pip-Boy wearable that comes with the collector's edition of Fallout 4 [32], and the cardboard backpack that transforms the player into a robot that is part of Nintendo Switch's Labo Kits [19]. In addition, the creators of last years' augmented-reality phenomenon Pokemon Go introduced Pokemon Go Plus [31], a wearable gadget for notifying players when there are new Pokemon around to catch. We can also consider virtual reality headsets and haptic/tactile gloves $[21,30]$ developed for these systems to be an additional special case of game wearables that is increasingly common.

Still, we would assert that most current-generation wearables are not living up to the full potential of such devices. Recent commercial examples are primarily used as companion interfaces [32] or superficially integrated without exploiting the game design opportunities possible with wearables. They are optional and their existence does not affect the game world, the six elements of game mechanics [46] or the ludic involvement [10] of the player, and therefore they do not deeply contribute to meaningful play [45]. For example, the PipBoy that comes with Fallout 4 is a gadget to which you can attach 
your smart phone and check character stats and inventory. While this device helps to bring the player closer to the fictional character on the screen, it does not meaningfully impact gameplay, to the disappointment of some players [32]. Similarly, Pokemon Go Plus does free players from continuously looking at their phones while wandering around, but does little else to augment or enhance the core experience of the game. In contrast, Nintendo Switch's Backpack turns into an embodied controller that allows players to use actions such as punching, crouching, walking or wearing/taking off a helmet. However, this example is heavily dependent upon screenbased interactions (the Switch device is embedded in the cardboard backpack and the interactions use the device's screen). The digital content of this gameplay has been criticized due to the lack of variety in game design elements [19]. We believe that current examples that incorporate wearables as a part of game play still do not fully exploit the design potential of wearables for game and controller design.

How can we improve the design of wearables for gameplay, to take full advantage of this potential? When it comes to academic research, although there are research projects that have focused on wearability and usability issues such as comfort [36] and ease of use [36], or that have evaluated appropriate technologies that can be used in wearables for games [5], we have found no in-depth investigation that examines wearables from a designerly point of view, to help researchers and practitioners best use these devices' capabilities to create rich and satisfying gameplay.

The three authors of this paper combined have extensive experience in designing and developing wearables that are deeply integrated into gameplay. Each has published peer-reviewed papers about gameplay wearables they have developed, as well as shown the accompanying games at public festivals and other peer-reviewed play venues. Working from our collective experience base, we have constructed a framework that characterizes key considerations in the creation of wearables for gameplay. This framework is built on three planes: performative, social and interactive. The performative plane focuses on qualities that wearables bring to game design that are also present in theater and costuming. Because wearables are worn on the body, they can be crafted as costumes that evoke fictional characters, thus fostering players' immersion in the imaginary world of the game [50]. The social plane focuses on how wearables invite players to socially interact with each other, for example inviting interdependence [22]. The third plane, interactivity, characterizes how different input and output modalities can be designed for wearables and how they can be incorporated in the game design.

In this paper, we use four wearable gameplay projects Hotaru, Magia Transformo, WEARPG and Social Wearables for LARP (SW4LARP) as exemplars to present the proposed design framework. We then discussed the framework as a tool with which to analyze and extend the design of these projects, to help show its utility as a thinking tool for others interested in the design of wearables for play.

\section{BACKGROUND}

\subsection{Wearables for Games}

Wearable controllers have been adopted by pervasive games research and used in many game applications. Mister X [2] was one of the predecessors of such games, and uses location data of players as a game mechanic by incorporating head-mounted devices as a gaming apparatus. Another early example is Human Pacman [12] which turns the physical world into a digital board with the help of backpack computers. These projects are relevant examples as they utilize wearables for providing mobility. Additionally, Berkowvky et al. proposed a wearable device as a companion interface for an exertion game which supports daily physical activity [4]. Apart from these examples, previous studies also considered social interaction through playful wearalbes. For instance, Nunez et al. developed a bracelet that hosts a digital pet which grows as children engage with each other socially [42]. This project uses simple light and vibration feedback for facilitating social activities and also lets the teacher monitor the social activity of children. A similar approach is followed by Firefly [52] for situating social interaction and collaboration between LiveAction Role Playing Gamers (LARP). As an extention of sociality, proximity between players were also exploited. An example project for this utilization is TagURit [11] that utilizes garments with embedded e-textiles for forming a proximity-based pervasive game. In this sense, these projects put the spotlight on how wearables can afford social interaction between players and shape proximity. Gauntlet [36] and Thumin Glove [53] are also game wearables which was developed for LARP games and are integrated in the game story and the mechanics. Different from Firefly, their focus is on adding new mechanics to games such as finding hidden objects with the help of haptic feedback. These projects makes valuable contributions by reporting the player feedback about the usability of the wearables and effects on game related concepts such as "seamlessness". Still, they do not contain a comprehensive analysis of the effects of wearables as artifacts on player experience.

Serious games also benefit from wearables while gamifying target activities. Freeman et al. [14] implemented a bracelet for helping visually impaired children move and play independently. One other project from Koskimäki et.al. introduced a wearable sensor based approach for encouraging the daily activities of young males [28]. RAPAEL [48] is another wearable technology for the rehabilitation of people suffering from brain diseases by aiming at turning mundane activities into exciting ones. Klann, in his study, used a participatory design approach for creating wearables for the training of the Paris Fire Brigade in a gamified way [27].

These examples show that wearables are used by rich variety of games in different genres that have different aims and target groups. Research projects curated in this section highlight different characteristics of wearables and demonstrate that wearable interfaces are convenient and promising for facilitating various types of interactions styles and adaptable to different game genres and mechanics. However, wearable artifacts in these projects are results of the needs for specific functions such as mobility, integration of body or movement. They did not investigate the design qualities of wearables and also did not consider how these qualities can further help towards the key experiences aimed by these projects. The design framework put forth in this paper contributes to the field by creating a tool and vocabulary that might be guiding and inspiring for the design of games that incorporate wearables.

\subsection{Design Spaces for Wearables and Games}

Design space is a term shared by many studies that focus on different characteristics of design features of interactive systems or artifacts. 
A commonality among these studies is that they use the term design space to create a framework that will help researchers to analyze the design of existing systems and create a vocabulary that can lead to systematic discussion. While some of these focus more on technical issues $[1,44]$ such as a communication method or the battery life of a system, others have focused on issues such as human factors [16, 35], interaction modalities $[18,29,40]$ or form-related issues of artifacts $[18,35]$. In terms of wearables, the work of Gemperle et al., Designing for Wearability [16], is a comprehensive framework that proposed 13 guidelines for designing wearables. This work focused on the design of the artifact itself by providing design directions on topics such as placement of wearables around the body, size variations, comfortable attachment, and proprioception. It also mentions that wearables can be subject to passive and active interaction, and that interaction with these devices should be simple and intuitive. On the other hand, Anliker et al. [1] takes a technology-focused approach to the design of wearable architectures by focusing on technical features such as battery capacity. Martin et al.[35], extending the technicality, also examines form and interaction-related issues such as human-movement and draping of clothes, as well as technical issues such as power consumption or networking capabilities of electronic textiles. Other than that, Moen used wearables in her work that focused on movement-based interaction in a non-technological and human-centered way [37]. In this work, she defined design concepts and guidelines that were adopted from the modern dance discipline. Although this paper is a solid example of defining a design space focusing on interactions and experiences, its focus was on movement and used wearables as a mediator tool.

Besides the wearables field, defining design spaces for shaping the design of games is also a common practice in game research. One well-known design framework in game research is the mechanicsdynamics-aesthetics (MDA) framework [20]. This framework proposes a vocabulary for game design analysis that breaks game design into three facets. Mechanics are rules and algorithms that establish the underlaying mechanisms of games, Dynamics are interactions between players and the game that arise through the interplay of mechanics as they player engages the game. Aesthetics are the emotional experiences a player has, that arise out of the play experience. Game designers are encouraged to think about all three facets of game design as they work, moving outward from mechanics to the end experience for the player, and using aesthetic goals to help them shape and tune mechanics and dynamics. MDA is an example of a framework focused on the analysis of the experiences and interaction activities in a system rather than the technical or physical properties. Another example is a recent framework put forth by Mueller et al. [39], which extensively covers a diverse set of bodily interplay types among players through three categories: coupling, physical space and virtual space. This framework is also a strong example that shows how defining a design space can facilitate discussions focusing on game experiences and help designers to extend current game structures. Bergström and Björk also put forth a comprehensive design space for analog games that are augmented by the integration of computation [3]. This design space introduces eight spectrums which can shape the design of computer augmented games and compares computer augmented games to both fully digital and fully analog games.

The wearable design frameworks mentioned in this section mostly focus on the artifact itself and they do not delve into the experiences and interactions that can be facilitated by wearables. When it comes to game design frameworks, we can see that their aim is closer to our design framework as they focus on the activity and player experience. However, to the best of our knowledge, the design space of wearables for play was not examined by previous game research studies. Therefore, we believe that the design framework defined in this paper will be beneficial for both the game design and wearables fields. In terms of game design, it guides and inspires design activities that focus on game mechanics and game experiences such as engagement and immersion. It also casts light on interaction styles and user experiences that can be afforded by wearables in other fields of $\mathrm{HCI}$.

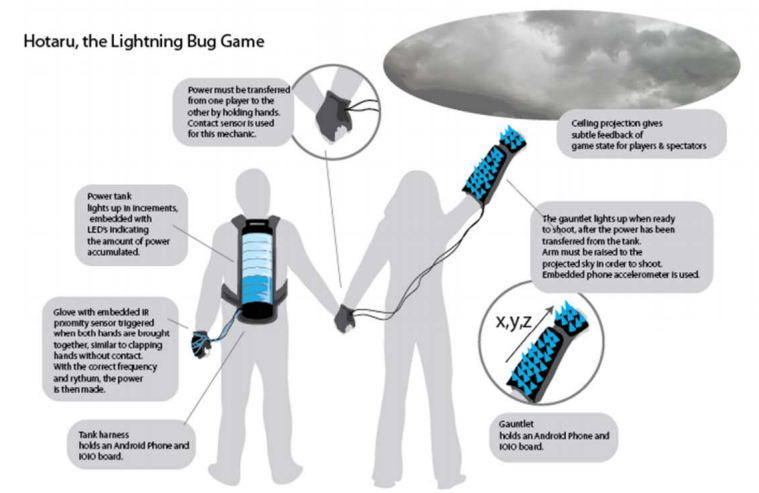

Figure 2: Diagram showing gameplay elements and mechanics of Hotaru

\section{PROJECTS}

We've selected four of our own wearable play projects for inclusion here. Each exemplifies a particular aspect of wearable play that we incorporate into our framework. These four projects produced knowledge through a combination of iterative design, rigorous user studies, and expert review with professional game designers. The guidelines and design knowledge that emerged from WEARPG show how wearables can be incorporated into long term narrative games. It also shows how wearables can incorporate diverse interaction modalities into long-term games with complex mechanics. Hotaru highlights how wearables can support interdependent play. Hotaru's wearables work as a bridge between the physical and imaginary worlds. Magia Transformo focuses on theatrical and performative aspects of wearables. It demonstrates how full body costumes can encourage role-play and identity exploration. Finally, SW4LARP, introduces the affordances of social wearables for play. Expanding on the dynamics of interdependent play introduced in Hotaru, SW4LARP puts forth a comprehensive theoretical analysis of the social features of wearables beyond bodily interpersonal interaction. In the next section we explore each system in greater depth.

\subsection{Hotaru}

Hotaru (Figure 1 - A) is a wearables-based game designed and built by artist Kaho Abe, as part of a multi-year research collaboration with Katherine Isbister, that explored the potential of costumes as game controllers. Hotaru is a two-player game-each person wears a unique device with complementary features. The premise of the game is that the players are the last two fireflies on earth, and must work together to keep back an evil fog. The first player wears a backpack (see Figure 2) as well as two gloves. The second player wears a glove 
on the left hand and a gauntlet on the right hand. Gameplay requires the performance of special gestures. The first player must move their hands together and apart (like trying to make a ball or to generate chi), and this motion causes the backpack on their back to slowly illuminate. When it is fully illuminated, the players can hold hands and 'transfer' the energy to the second player. That player's gauntlet will slowly illuminate. When it is fully illuminated, the second player can raise their arm, 'releasing' the energy and causing a powerful sound. Players work together to gather and release as much energy as they can, to defeat the evil fog. Hotaru has been shown at CHI, TEI, IndieCade, and Play Publikum in Poland, among other venues. Apart from the iterative tests in the design process, 62 users formally took part in the evaluation that investigates the effects of the game on connection between players was also published at $\mathrm{CHI}$ [22].

\subsection{Magia Transformo}

Magia Transformo (Figure 1 - B) is a wearable mixed reality experience designed by Tess Tanenbaum, Natalie Nygaard, and Ke Jing at UC Irvine's Transformative Play Lab. Three players take on the roles of apprentice witches, selecting magical cloaks and hats (Figure 3) to transform into different characters, using the costumes to embody the different covens and affiliations present within the world. Gameplay is divided into two different phases: costume exploration where players assemble outfits and characters, and spell casting where players dance around a magical cauldron holding interactive spell books and chanting magical words while performing ritual motions to discover different magical abilities. Magia Transformo has been presented at CHI PLAY [24] and demonstrated at IndieCade (with the participation of around 65 players), and is currently being expanded to include an Augmented Reality (AR) mobile game component that expands on the magical world and provides a richer narrative context for the ritual experience of the initial prototype.

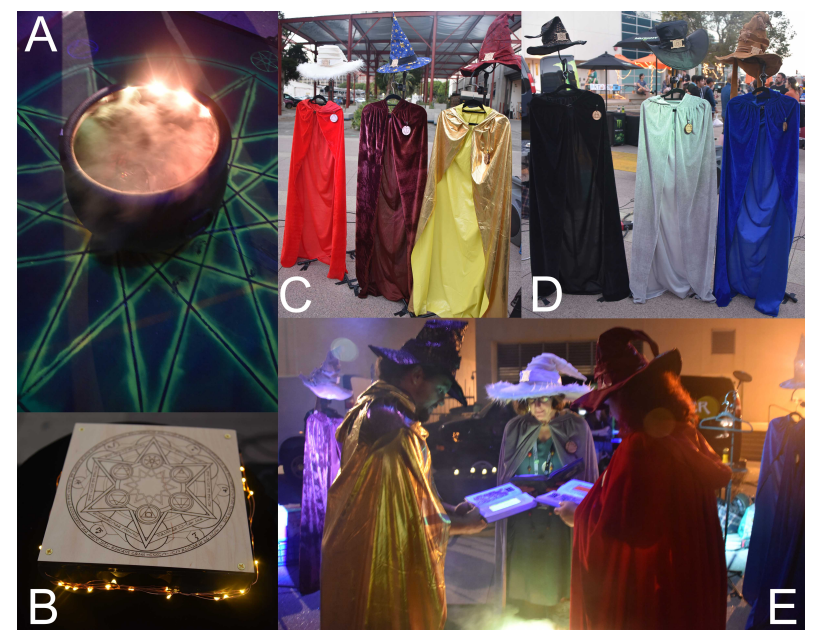

Figure 3: Magia Transformo's elements: (A) The Magic Cauldron, (B) The RFID Altar, (C \& D) The Costumes and Magic Symbols, (E) The game in action

\subsection{WEARPG}

WEARPG (Figure 1 - D) is an augmented and movement-based tabletop role-playing game designed by Oğuz Turan Buruk, İsmet Melih Özbeyli and Oğuzhan Özcan in Koç University - Arçelik Research
Center for Creative Industries. It incorporates Elemental Gauntlets which are arm-worn devices and an augmented die, Luck Stone (Figure 4). Elemental Gauntlet has a light-disc and haptic motors and is the interface which facilitates the movement-based games. Moreover, it also has a tangible interface for character creation in which players can attach different types of element stones for creating their imaginary characters with different features. It is comprised of hexagonic units which allow players to built their own Elemental Gauntlets and customize it as they level up. Luck Stone, besides having a role in several movement-based mini-games, is the randomization device and the tangible element of WEARPG game system. In WEARPG, players need to play movement-based mini games with the help of the Elemental Gauntlet and the Luck Stone. Success in these games affect the probability of the Luck Stone, meaning that if the player is successful in the mini-game, green faces of the Luck Stone will increase. There are seven different types of mini-games in the WEARPG system which are designed for different events that can require different character skills such as power, reflex, precision and concentration. WEARPG is a result of an extensive research through design process which includes a participatory design workshop, iterative prototyping, wizard-of-oz testing and final play testing. Development of the game took around two years with the participation of RPG players, game masters, cosplayers and experts in the fields of fashion and interaction design. In total, 53 participants contributed to design process of WEARPG. WEARPG has been presented, demonstrated and awarded in venues such as CHI [9], CHI PLAY [8] and DIS [7].

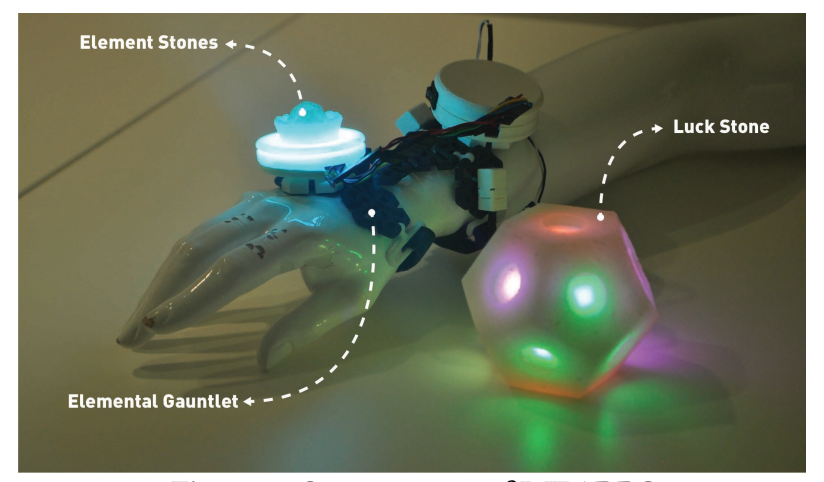

Figure 4: Components of WEARPG

\subsection{SW4LARP}

The wearable devices from the SW4LARP project were developed in collaboration with LARP designer Shoshana Kessock for use in the Battlestar Galatica: Tales of the Rising Star (BSG) Live Action Role-playing Game (LARP). In a research-through-design process, a subset of Katherine Isbister's lab group led by Elena Márquez Segura developed two final prototyped devices -one to help players display their current health status, and another to help them communicate their current mental energy level. Both devices were built on the infrastructure of the Adafruit Circuit Playground, a consumer-grade device aimed at the do-it-yourself (DIY) maker community, with additional components and custom software. For the sake of brevity, here we focus on just one of the two prototypes-for more detail, see [34]. The health status prototype (see Figure 1 - C, pendant) was worn around the neck like dog tags (something that BSG players 
already used in their costuming). This device included an additional neopixel and a vibrating mini motor disk. The central pixel ring on the device was a visualization of a player's health level-lots of glowing green indicated a health score between 6 and 10, yellow meant fair health (between 5 and 3 points), and red indicated critically low health (less than 3 points). If the player's health got to zero, all pixels would light up and flash red. Players set their health value themselves, by using two small buttons on the face of the device to raise and lower their own health score as things happened to them in-game. The motor built into the health device had two modes, which could be toggled using a built-in switch: a heartbeat mode, which vibrated at different speed depending upon the health of the player (less health, more widely spaced vibrations), and a navitation mode. In the latter, the motor vibrated when the player raised or lowered their health score. At the same time, an onboard speaker would play a tone with a frequency that corresponded with the health value (higher frequence for higher health). The additional external neopixel allowed players to indicate whether they were okay with physical contact or not (red=no; blue=yes). Both prototypes were tested in the BSG LARP experience, held at Dexcon 20 in New Jersey. Test included 36 prototype used by 19 Player in an almost 100 player LARP setting. Players made creative use of the health status prototypes, using them in the manner intended by the LARP designer and the device creators, and also, improvising additional functions of the wearables in support of twists and turns of the action that took place as the narrative progressed. For more detail about this, see [34]

\section{DESIGN FRAMEWORK}

These four projects illustrate the range of wearables that the authors have built in service of gameplay, and are examples of research through design [56], in which the authors have engaged in expert hands-on design and development as well as conducted rigorous player experience studies and observations. This intellectual process of creating theoretical knowledge through design practice was also previously framed by the "reflective practitioner" [47] concept of Schön. Research through design is an effective way of converting the tacit knowledge of a designer (as discussed in Nigel Cross's "designerly ways of knowing" [13]) into a form from which other researchers and designers can also benefit. One common way to reflect this design practice in relation to research is utilizing Annotated Portfolios $[6,15,33]$. These map theory and general reflections onto particular artifacts and their use characteristics. Following this strategy of sharing generalizable knowledge from specific design instances, the design process of the projects included in this paper and their unique contributions to the field can be found in the "Projects" section of this paper, and far greater detail can also be found in the publications cited for each project. Furthermore, each dimension of the framework is explicitly mapped to the features of these projects in the following sections. Using these four projects, as has been done in similar papers in the HCI-game subfield [3, 38, 39], we created a design framework for playful wearables.

There are ten components in this design framework (see Figure 6), each categorized under one of three 'planes': performative, social and interactive. Each of these three planes defines design directions and opportunities that are particularly relevant for wearables, that can help lead to novel interaction styles, game mechanics and experiences. Components of the performative plane are focused on the ways that design choices for wearables can shift games between being more "Imaginary" or more "Immersive". By Immersive, we mean character identification and a more refined game world. By imaginary, we mean game characteristics that are purposefully incomplete and left to the imagination of players. The social plane considers the range between "Tight" and "Relaxed" social interactions between players. By tight interactions, we mean activities such as bodily contact or interdependent play; by relaxed, we mean less tightly coupled and close interactions such as verbal conversations or gesturing/signing from a distance. In the interactive plane, wearable design choices can facilitate a range from "Peripheral" to more "Artifact-Oriented" interactions. Peripheral interactions include embodied or tangible interactions likely to be visible to others and performed in relation with and awareness of the environment. By artifact-oriented interactions we mean more digital and embedded interaction modalities, which can, for example, facilitate hiding private information. Each plane includes sets of spectrums in which games are positioned to determine their positioning in one of the two-sides of the planes (see Figure 5 and Figure 6 for examples) Though each component is not strictly orthogonal to the others, they were selected on the merits of providing value when considered independently from one another to generate new insights and ideas for the design of wearables to enable gameplay. Alteration of one dimension could lead to changes in how a project would be characterized on another dimension. Characteristics of design decisions can place wearables in different areas, as can be seen in Figure 5, and experimenting with dimensions can take one project and shift it towards the opposite direction, as in the Hotaru Example. This will become more clear as we move through the re-imagining of the games described in this paper through the lens of the framework in the "Discussion" section.

To create the framework outlined in the next section, we engaged in iterative discussions in a series of both in-person and online meetings, as well as ongoing email correspondence. Buruk began the analysis with an initial set of design facets that he gleaned from a close read of each of the four projects. Isbister and Tanenbaum provided critical reflection on these facets, and the group shifted and reframed some of them through discussion, in an iterative process. The iterative revisions to the framework can be divided into 'high level' and 'low level' adjustments. At the low level, each dimension was refined in terms of conceptual definitions. A concrete example can be given for the "costumes-accessories" dimension. In the first version, the main difference between costumes and accessories referred only to difference in their size and the portion they cover on the body. In the final version, however, possible effects of costumes and accessories on self-expression and representation to onlookers is also discussed. At the high level, we reframed the relationship between the three main categories. In the beginning, we considered these as "spheres", mere categories which are weakly connected to each other and to spectrums. In the last version, our ideation on the relations between these dimensions and categories resulted in an analytic tool and defined a 3D space where the positioning of games in each dimension corresponds to a location (Figure 5.) The final version of this framework successfully captures the key elements of the four projects described in the paper, and has also been exercised upon a commercial game example (discussed later in the paper) with success (see Figure 5 and 6 for the positioning of games in design space and each dimension). 

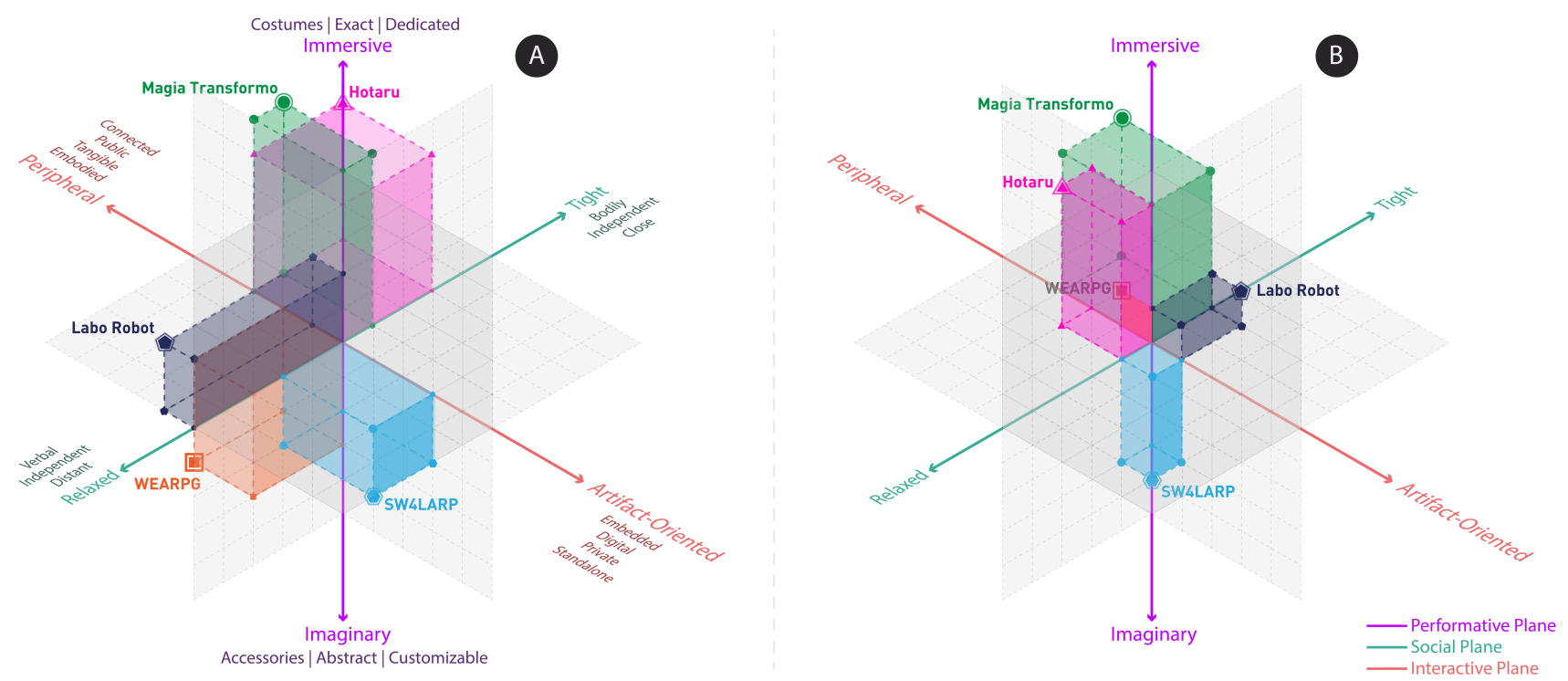

Figure 5: A) Current Versions of Games in the Design Space, B) Extended Versions (see Discussion section)

\subsection{Performative Plane}

The most immediately noticeable characteristic of wearables, as compared to other types of control devices, is the fact that they are worn. Thus, in the scope of play, contrary to most game interfaces, operational functionality is not the only strength of wearables. Wearables can also contribute to the play experience by offering players a chance to physically/visually transform into a fictional character, and/or to engage in performative self-expression. In this plane, we discuss how performative aspects of wearables can affect the design of the play experience.

4.1.1 Costumes-Accessories. Wearables can function as costumes that cover some or all of a player's body (Magia Transformo, Hotaru's backpack and decorative gauntlet) or can be worn on smaller, targeted parts of the body in the form of bracelets, pendants (SW4LARP), or gauntlets (WEARPG). When wearables are perceived more like a costume by players, they will have a bigger effect on the transformation of the player into the fictional being they intend to be. Moreover, when they cover a bigger part of the body, they are more visible to other players or spectators, which can also encourage players to act like their characters. Accessories, on the other hand, are traditionally designed to be worn more frequently on a specific part of the body in a variety of circumstances, each of which requires the particular operational functionality that the accessory provides. Accessories can be more suitable to game scenarios in which they need to be carried around or taken on/off frequently. For all of these reasons, the costume side of this spectrum may promise a stronger shift towards the imaginary world of the play, while the accessory side comes with advantages such as easy deployment and ease of re-use.

4.1.2 Exact-Abstract. The act of putting on something can be a ritual for transformation [25]. Once a wearable artifact is put on or attached to the body of the player, they can get closer to their fictional-self while loosening their connection to or focus on their real-self. In this sense, the appearance of the wearable artifact can affect the type of transformation players can experience. On the exact side of this spectrum, a wearable that is designed for a specific story or character may help players to identify themselves with a predefined character, or put them into an imaginary world whose boundaries are more visible, as in Magia Transformo or Hotaru. On the other end of this spectrum, abstract designs such as in SW4LARP or WEARPG may leave more space for the imagination of the players, and can fit more flexible stories.

4.1.3 Customizable-Dedicated. Similarly, customization is a feature that can feed different aspects of performativity. As the design of a

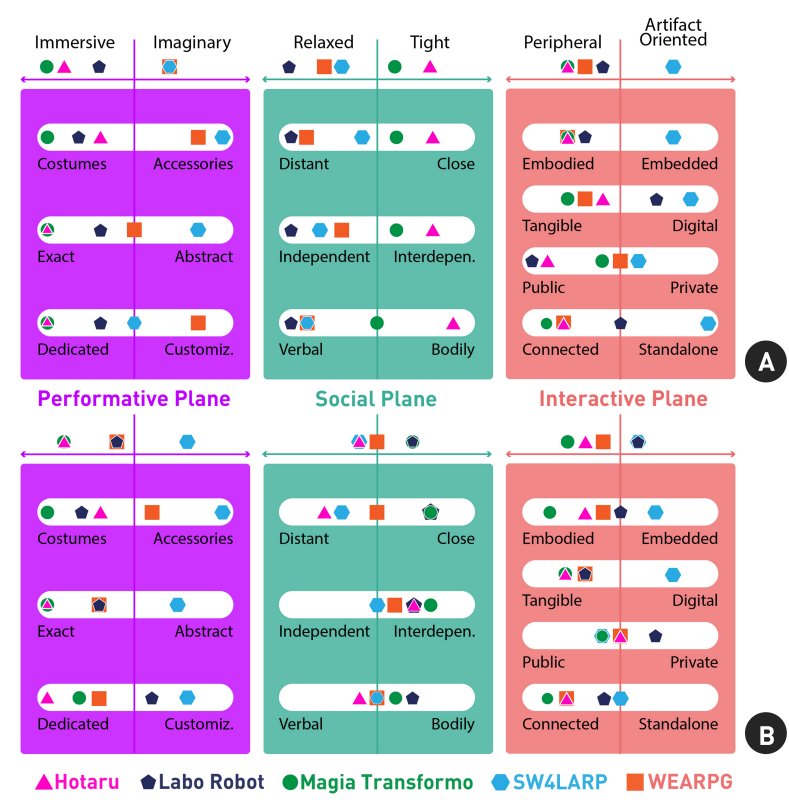

Figure 6: A) Place of the Current Version of Games in Each Spectrum, B) Extended Versions (see Discussion section) 
wearable shifts towards the customization side, players are granted the freedom of expressing who they want to be in the imaginary world. In case of WEARPG, players quite enjoyed customizing and creating their own gauntlets, and they put forth distinct designs. SW4LARP was repurposed in the LARP where it was deployed in creative ways that were afforded by the device's flexibility. However, motion or other types of body sensors may not work as intended if they are worn on different parts of the body or in different positions than they ought to be. Therefore, on the other end of the spectrum, dedicated designs can present more controlled and refined experiences, as in Hotaru and Magia Transformo This differs from the "exact-abstract" spectrum. While the former is concerned with how much semantic information is evoked by the designed wearable, the latter represents the extent to which the design anticipates and encouraged user intervention.

\subsection{Social Plane}

One of the unique aspects of wearables is their capacity to drive social interaction between players. Designers can craft wearables in ways that shape social behaviors among players. In this plane, we examine the design decisions that can lead to different social dynamics in the play experience.

4.2.1 Distant-Close. Wearables can be the source of both distant and close social encounters between players. They can help designers to shape proxemics [23] between players, without needing additional interface elements. For example, by utilizing touch interaction modalities as in Hotaru and SW4LARP, they can facilitate physical interaction between players through play. While the close side of this spectrum may lead to a game experience where bodies of two players actively affect each other, on the other side of the spectrum, wearables can also convey information that can be rendered visible to others from a distance. This also reflect to the research on spectator interactions which is a common concern in games for social interaction [23], especially by incorporating expressive and magical interaction styles [43]. Here, we refer to real spectators who are not actively involved in the game, as well as to active distant or inactive spectating players who are waiting for a turn. For example, in SW4LARP, players can change the color of the lights in their pendants to express if they are open to physical social interaction or not. Another example is the movement-based game play scenes in WEARPG, which other players enjoyed watching. Wearables are well positioned to affect the closeness or distance between players as interact, and to generate interesting spectacle for others.

4.2.2 Interdependent-Independent. Wearables can bring players closer through modalities such as interpersonal touch. They can also facilitate game mechanics which need synchronization of players, by giving immediate and visible feedback about player moves. In this sense, wearables are very suitable for evoking interdependent play experiences. For example, in Hotaru and Magia Transformo, players are heavily dependent on each others' actions, due to the game mechanics administered by the wearables. In Hotaru, the motion sensor in the glove and the visual display on the backpack let players coordinate their actions and physically connect them to each other. In Magia Transformo, Wizard Hats are tracked by a Kinect Sensor which assures that all players are in the right track. Still, wearables can also provide social engagement without making players explicitly dependent on each others' moves. In SW4LARP, for example, the Health Pendant does not use an interdependent mechanic, but can still facilitate social interaction. During the game, when players saw the different light statuses which represent the health level of players, they started conversations about the imaginary health condition of player. With WEARPG, although all players play movement-based games by themselves, the devices keep other players engaged with the game, since the movement-based mini-games and the visual feedback from the devices attracted the attention of passive players and created memorable moments. Therefore, wearables work well towards creating an interdependent play structure, yet they are also capable of maintaining social interaction between players through independent play.

4.2.3 Verbal-Bodily. Interdependence expedited by wearables usually comes in the form of bodily interplay. In Hotaru, Magia Transormo and in the case of the Mental Energy module of SW4LARP, the interaction between bodies of players is a critical element of the gameplay. On the other side of this spectrum, we can see how wearables can foster verbal role-playing elements in a game. In WEARPG and SW4LARP, we observed that wearables are great conversation starters that facilitate verbal communication between players. In the WEARPG case, we saw that players talked to each other to discuss and discover the so-called super powers of gauntlets as a part of their roleplay. Similarly, the Mental Energy modules of SW4LARP not only encouraged physical interaction, but also verbal interaction, such as commenting on other players' low mental energy and suggesting that they should rest for a while. As a design direction, wearables can lead players to get into physical and bodily social interaction with other players. But also, wearables can easily become attraction points in a game, and can work as conversation starter for verbal social play.

\subsection{Interactive Plane}

With wearables, designers can introduce many distinct interaction modalities to games. In this plane, we define the different interaction modalities and discuss the information structures wearables can support for play.

4.3.1 Embodied - Embedded Interaction. It is common to use wearables as a substrate for sensors that track body data such as GSR, EEG, motion, or heart rate. In addition to this, wearables create surfaces around the body that can support several different output and input types such as haptic or visual feedback. As an example for embodied interaction, WEARPG and Hotaru utilize integrated motion sensors for involving players in the game with their bodies. Different from these two, Magia Transformo uses hats and hoods as traceable markers that can be tracked by external sensors such as Kinect or RGB cameras. Towards the "embedded-end" of the spectrum, SW4LARP takes the opportunity to use the body as a surface for switches [50]. In this game, rather than using gestural interaction, users can use the props and swap between their functions by using buttons, switches and touch interaction. Therefore, besides the common usage style which is embodied interaction, designers should not also miss the opportunity to use embedded interaction modalities such as touch interaction, as they may be more comfortable in complex functions. 
4.3.2 Tangible - Digital Interaction. The human body is comprised of wide surfaces which can be exploited by wearables. As wearables are shaped according to body parts they are worn upon, they can have surfaces in different shapes that can allow physicality with additional tangible parts, switches or buttons. For example, in WEARPG, we could use the hexagonic base of the Elemental Gauntlets as surfaces where players could attach new parts to customize their props. Moreover, when different element stones are attached to the socket the appearance of the gauntlet changes as well as its game-related properties. Therefore, these surfaces can be used as a platform for tangible interaction. What is important here is that, as a result of the tangible interaction, it is very likely to see that the visual properties of the wearable may also change. These changes can also affect the performative plane, as they will alter how the fictional being of the player is represented. Other than custom tangible interaction methods, these surfaces can also be used for placing more traditional digital interaction devices such as displays or touch areas. For example, Hotaru adorns both the backpack and the gauntlet with LED-based illumination that gives feedback about the progress of players. We believe that the balance between tangible and digital interaction is an important design opportunity: digital interaction may easily facilitate conveying important information while the tangible increases physicality. Moreover, these modalities also affect the visual state of wearables in different ways that are closely related to the performative state of the wearables.

4.3.3 Public - Private Information. Wearables, by design, are suitable interfaces for embedding private and public information, as they use various surfaces of our body. For instance, information which is meant to be seen by others can be placed on a larger, more noticeable surface such as the torso or upper arm. The same information could be placed on back of the wrist, palm or inside of a robe, if it is meant to be concealed. In this sense, wearables can support all of the dimensions of Reeves' spectator's view framework [43] which are magical, expressive, secretive and suspenseful. Hotaru exemplifies the artful use of selective access to information, as only one player can see the information on the backpack, which is an important element for the gameplay. Similarly, in WEARPG, players can hide the light-disc inside their palms if they want to conceal information from other players. In addition, haptic feedback can be used to inform players about secret events in the game which should not be visible to other players, as is done in WEARPG and SW4LARP. On the other hand, SW4LARP and Magia Transformo communicate public information that can easily be seen by other players, such as health status or character types. We believe playing with the privacy of information supports a wide range of game mechanics and role-playing opportunities for designers.

4.3.4 Standalone - Connected. Wearable artifacts have different advantages on the two ends of this spectrum. On the standalone side, wearables become more suitable to play experiences that employ large areas, allowing players to move around freely without relying on a connection or a detection of another device such as a main computer or a Kinect Sensor. In that sense, props designed for SW4LARP take advantage of working in a standalone condition, allowing players to larp freely. On the other hand, wearable can introduce ways to connect bodily experiences to game mechanics. In WEARPG, interaction between the Elemental Gauntlets and the Luck Stone provides an experience where players see the effects of body movements directly on the Luck Stone and thereby in game mechanics. In Magia Transformo, wearables are connected to a Kinect Sensor and an RPG camera by their different colors, and similar to WEARPG, they provide a connection to the Altar in the middle. Another connected interaction model is used by Hotaru. Different from WEARPG and Magia Transformo, Hotaru uses connectedness for facilitating interdependency and physical social interaction between players. In a nutshell, "standalone" features provide more freedom, which can allow players to move around more comfortably, while connected modalities promise richness in terms of game mechanics.

\section{DISCUSSION}

\subsection{Using the Framework}

We believe that the benefit of our design framework is twofold. First, it provides a vocabulary for discussing and analyzing the current structure of games, helping to highlight existing strengths and potentials. Second, it can be used as an experimental tool for uncovering new dimensions and future directions for designers to extend their games. Here, we will discuss different ways of using this framework by applying it to games presented in this paper, an external commercial example (Nintento Labo Robot Kit [41]) and an external non-game wearable research study (Social Textiles Shirt [26].)

To demonstrate the effectiveness of the framework, we have deployed it in several different contexts. We use the framework as an analysis tool of the current state of SW4LARP to show how to use the vocabulary it provides. For analyzing WEARPG, we take a more critical approach to recognize the missing opportunities in the design and recommend extensions in this direction. We also see this framework as an inspirational tool with which designers can experiment on their design choices, and the extension of Hotaru is an example of this type of usage. Lastly, we use it to further improve the existing features of Magia Transformo. For the external projects, we demonstrate how our framework can be useful to ideate on new game mechanics, while still remaining true to the design philosophy of the existing product in the Labo Robot Kit example. Considering the non-game example (Social Textiles Shirt), we show that our framework is not limited to gaming applications, but also can be useful for designing and extending playful interactions in other contexts.

5.1.1 SW4LARP. For SW4LARP, we use the framework only for analyzing the existing structure of the game design. Our purpose here is to present how the framework will enrich the definition and the discussion of the game design. SW4LARP exhibits the ability of wearables to enhance role-playing aspects when they are placed in the "Imaginary part" of the performative plane. During the gameplay of Battlestar Galactica LARP, players included the devices in their own improvisations, by reacting to the feedback of the Health Modules in ways that were unanticipated by the designers. Moreover, it also shows that wearables can trigger verbal interactions between players by representing information that is meaningful in the game context (staying close to "verbal-end" of the spectrum). Considering these, SW4LARP is a good example how the "Relaxed" side of the social plane and the "Imaginary" side of the performative plane can work in games. In the Interactive Plane, it also demonstrates that wearables are not limited to the category of devices that are subject to embodied input, but can also include embedded modalities, such as switches or buttons, as also previously discussed by Tess and Karen Tanenbaum [50]. 
5.1.2 WEARPG. Here, we used the framework as a critical tool to discover the missed opportunities of the current game design structure and extend it to realize the intended key experiences. We believe that WEARPG's customizability and mobility are important for and relevant to the nature of RPGs. Still, walking through our framework, we see the opportunity for providing more refined visual qualities to players in the next iterations. We believe that a better balance between customizability and a dedicated look can result in increased immersion. By providing pre-designed parts specific to different types of characters, fictional worlds or stories, we can establish such balance. In the social plane, we believe that modifications that would allow more close and bodily interactions with other players would be an invaluable addition. That might take the social interactions in RPG to a new level. Moreover, this could even change the physical game environment in a way that allowed players to form bodily contact during the game. Bringing the game closer to the "inderdependent-, close- and bodily-end" of the spectrum could also result in new RPG mechanics. A simple example is a scenario in which players play movement-based games that require them to synchronize the timing of their body movements in order to combine different types of attacks into a collective attack-move with a stronger impact. Finally, for the interactive plane, we can improve the embedded outputs. Although, not using a display was a design decision for preventing gauntlets from distracting players during gameplay, equipping gauntlets with a feedback mechanism that informs players about the levels of specific character features, such as power or quickness, could add to both game experience and interaction between players.

5.1.3 Hotaru. While extending Hotaru, we will follow an experimental approach to see how playing radically with the different sides of the planes can result in interesting new gameplay possibilities. We articulated that interdependence by close bodily interactions is a strong part of Hotaru, and due to this, it is positioned strongly in the "Tight" side of the social plane. Here we ideate on a version of Hotaru that could be positioned in the relaxed part of the spectrum, while keeping the interdependent features. Thus, we will consider the "distant" and "verbal" parts of the spectrum and also add "tangible" elements to facilitate new gameplay elements created by this decision. The gauntlet in Hotaru has spikes that light up as energy is transferred from one player to another when they hold hands. These spikes could also be tangible object that are scattered and hidden in the game environment. One of the players would need to collect them and bring them back to the Gauntlet bearer to place them on the gauntlet. While collecting these objects, the Gauntlet Bearer would guide the collector due to vibrations s/he feels as the collector gets close to the artifacts. We believe that this addition might even render the hand-holding activity more interesting as it would occur less frequently. These additions would place Hotaru in the "Relaxed" part of the social plane, while still maintaining interdependent aspects.

5.1.4 Magia Transformo. In this re-envisioning exercise, we will demonstrate how the framework can be used to boost and polish existing elements of a game. We might modify Magia Transformo's design to add new customizable elements, that might allow players to invest more of their own personalities into their experience of the game. We also see opportunities to increase "interdependency" between players by bringing their physical interactions closer. By increasing player contact (through mechanics around hand holding, or more sophisticated partner dancing) we might produce a more powerful social connection between players. Implementing these kinds of mechanics would require the creation of new wearables with additional sensors embedded within them, but would allow us to detect more complex player behaviors, allowing for deeper gameplay, and a more pronounced skill progression for players. All in all, shifting Magia Transformo towards the "Tight" side of the social plane may intensify the existing game atmosphere which is formed by the contribution of all players. Moreover, by pulling it towards the "Imaginary" side of the performative plane with increased "customizability", players' role and place in this magical world can be more believable as they feel their personal impact.

5.1.5 Nintendo Labo Robot Kit. While ideating on the Labo Kit, we will remain true to the current working mechanism and Nintendo Switch's proposed values to the gaming console market, such as mobile, co-located multiplayer features. Labo Kits promote do-ityourself culture and promise a wide range of customizability options, by allowing players to access and program controllers via a simple and visual programming interface. In this sense, Labo Robot Kits could be located closer to the "customizable-end" of the spectrum as the materials and design philosophy of Labo are very suitable for this. By creating different types of folding lines, players could create different designs, and these designs could also be read by controllers to change the character in the digital part of the game. In addition, the current design uses one of the controllers for detecting if the visor is on or off, which does not have a big impact on gameplay. By taking the opportunity of an additional controller, Labo kits could use the second controller for facilitating social interaction, by integrating a second player, as this was also one of the promotion points of Nintendo Switch. To foster "interdependent" and "close" interaction, the second controller could be placed onto the backpack and recognize different button combinations or tangible stud-like cardboard objects as different weapons for different kinds of enemies. While one of the players would decide the weapon type by inserting tangible parts to the weapon module, the other one could focus on aiming and shooting. This would carry Robot Kit towards the "Tight" side of the social plane, and also be a more effective way for using the second controller. This weapon module could even be worn on the arm and controlled by the same player, in case the player wanted to play in solo mode. As this addition also create a "connectedness" which is tethered to game mechanics, Labo Robot shifts towards "connected" side of the spectrum. To summarize, as examined through the framework, we believe that carrying Robot Kit from the "Peripheral" and "Relaxed" sides to the "Artifact-Oriented" and "Tight" sides could create new gameplay opportunities, favoring efficient use of controllers and the design philosophy of Nintendo Switch favoring sociability.

In the process of analyzing our own games, we found that the design framework helped us to develop more grounded and concrete observations about the work that particular design decisions about the wearables was doing for players. Using the framework also helped us to envision alternative paths and future opportunities for these experiences, using the three planes of performativity, social interaction and interaction modalities. We were also able to use the framework to evaluate a commercial game design, showing that it can extend and encompass designs outside our own practice. 
5.1.6 Non-Game Example: Social Textile Shirt. Up to this point, we have focused on analysis of game-based wearables. We believe that the framework can also be of value to HCI practitioners outside of games, who are designing wearables-based experiences. For example, the Social Textiles Shirt [26] project aims to facilitate social interaction between wearers of this product. The shirt notifies the wearer when other people who wear the same type shirt are around. Once they find each other by recognizing themselves from the shirts they wear, they shake hands so that the shirt will reveal some information based upon the context and their acquaintance level. For example, in one of the scenarios, when two students in a university orientation event who wear different colors of shirts shake hands, the shirts reveal their majors.

Although we could conduct a more thorough analysis of this project by considering all of the planes, we will focus on only the social plane due to limited space. Apparently, this shirt is designed for a "close" and "bodily" interaction, as it aims to encourage users to shake hands. It also gives hints about verbal social interaction, as the information about majors that it provides could help to start a conversation. We can also say that it is a little bit shifted towards interdependent interaction, since it relies on the two users shaking hands. Considering the social plane as a point of iterating the design, we believe that attending to the "verbal-end" of the "verbal-bodily" spectrum could yield promising results, since this project aims to be an ice-breaker between newly acquainted people. In this case, we could imagine using a symbol or a clue that is related to the major, instead of revealing the name of the major immediately. This might lead to a longer conversation in which the two wearers try to guess each other's department. This conversation could even get longer, if the symbol or clue is something worth talking about. Even this simple alteration could enhance the key experience intended by the project. Similar experiments can also be done by walking through the other dimensions of the social plane, and these might uncover scenarios in which different types of social dynamics are present. Drawing upon this quickly sketched example, we propose that our design framework could be useful for the design of wearables in realms outside of games, such as smart textiles, fashion, VR/AR research, and so on.

\subsection{Limitations}

We believe that the proposed framework encompasses a synthesis of the current design space around wearables for play. However, this is still an emerging area, and so there are few fully-realized examples within the commercial and non-game spaces. This limits our ability to ground our analysis in a broader technological ecology. The four research prototypes discussed within this paper cover a lot of the possible design space, but without more examples to consider, we acknowledge that our framework represents a first big step towards a comprehensive theory of wearable play, but is likely to need expansion at some future point. We argue that this framework has relevance to designers and theorists of wearables, but as is the case with much research through design we make no claims to experimental validity or reproducibility of results [56]. As new systems are built and released, we expect our framework to evolve. In parallel to this, we also plan to bring this framework to designers and developers of playful wearables, and to further refine the dimensions and planes based on their feedback. Our role in this case is to communicate the design process and the methods used in a clear way, to allow other researchers to follow a similar pattern. The design process of projects included in this paper were thoroughly explained and can be followed in their related publications. We also highlight that utilization of this framework does not guarantee a "better" design, as design is by nature subjective, and a "better" design heavily depends on the context. However, we can clearly assert that our framework is helpful for guiding designers towards a "right" design [56] by revealing the unexplored playful design opportunities provided by wearables. Our framework is the first step to better interpret wearables and fully exploit their capabilities while designing playful experiences.

As mentioned in the background section, there are similar frameworks and defined design spaces for both wearables and games. The scope of our framework is defining playful interactions and experiences that can be provided by wearables. However, we are aware that wearables have also other critical qualities such as comfort, fashionability or usability. Our framework is intended to extend, but not replace, these existing approaches. Specifically, after defining relations between the playfulness and the wearables, designers should also look for Body Maps [54, 55] to analyze the ideal body parts to place wearables upon, Wearability Guidelines [16] to design ergonomic and usable wearables, Fashonability studies $[17,51]$ to design towards self expression, aesthetics and social acceptance. Game design frameworks such as MDA [20] will also be useful to analyze game actions, mechanics and experiences created after the effective integration of wearables by using our framework.

\section{CONCLUSION}

In this paper, we put forth a novel design framework that focuses on the integration of wearables into play experiences. We based our framework on three planes: performative, social and interactive. We were motivated do create the framework because of increased interest in wearables for games and play both in academia and industry. Considering the limitations of current designs of wearables for play, we believe that such a design space can provide value to the community, casting light on further design opportunities that wearables can deliver.

This design framework was developed based on game projects, however it may also be useful for wearable designers in other fields such as smart textiles, VR/AR, and social wearables. Contrary to design frameworks developed for the design of wearables, our framework highlights the transformative experiences that are tied to selfexpression of wearers, social interaction activities and experiences that can be evoked by these interactions, and variety of interaction design modalities wearables are capable to administer. Therefore, we believe that designers both in and outside the game design field could benefit from it, both as a retrospective analytic tool, and also as an exploration instrument during their ideation stage.

\section{ACKNOWLEDGEMENTS}

This publication is partially funded by H2020 Marie SkłodowskaCurie Action, grant number 833731 - Designing and Developing Wearables for Virtual Reality Environments with a Research Through Design Process - WEARTUAL.

\section{REFERENCES}

[1] Urs Anliker, Jan Beutel, Matthias Dyer, Rolf Enzler, Paul Lukowicz, Lothar Thiele, and Gerhard Troster. 2004. A systematic approach to the design of distributed 
wearable systems. IEEE Trans. Comput. 53, 8 (2004), 1017-1033.

[2] Stavros Antifakos and Bernt Schiele. 2002. Bridging the gap between virtual and physical games using wearable sensors. In Wearable Computers, 2002.(ISWC 2002). Proceedings. Sixth International Symposium on. IEEE, 139-140.

[3] Karl Bergström and Staffan Björk. 2014. The case for computer-augmented games. Transactions of the Digital Games Research Association 1, 3 (2014).

[4] Shlomo Berkovsky, Mac Coombe, and Richard Helmer. 2010. Activity interface for physical activity motivating games. In Proceedings of the 15th international conference on Intelligent user interfaces. ACM, 273-276.

[5] Christian Bertelsmeyer, Erik Koch, and Alexander H Schirm. 2006. A new approach on wearable game design and its evaluation. In Proc. Netgames '06. 1-6.

[6] John Bowers. 2012. The logic of annotated portfolios: communicating the value of'research through design'. In Proceedings of the Designing Interactive Systems Conference. ACM, 68-77.

[7] Oğuz Turan Buruk, Ismet Melih Özbeyli, and Oğuzhan Özcan. 2017. Augmented Table-Top Role-Playing Game with Movement-Based Gameplay and ArmWorn Devices. In Proceedings of the 2016 ACM Conference Companion Publication on Designing Interactive Systems. ACM, 289-292.

[8] Oğuz Turan Buruk, İsmet Melih Özbeyli, and Oğuzhan Özcan. 2017. WEARPG: Movement-Based Tabletop Role-Playing Game with Arm-Worn Devices and an Augmented Die. In Extended Abstracts Publication of the Annual Symposium on Computer-Human Interaction in Play - CHI PLAY'17 Extended Abstracts. ACM, 639-646.

[9] Oğuz Turan Buruk and Oğuzhan Özcan. 2018. Extracting Design Guidelines for Wearables and Movement in Tabletop Role-Playing Games via a Research Through Design Process. In Proceedings of the 2018 CHI Conference on Human Factors in Computing Systems. ACM, 513.

[10] Gordon Calleja. 2011. In-game: From immersion to incorporation. MIT Press.

11] Sylvia H Cheng, Kibum Kim, and Roel Vertegaal. 2011. TagURit: a proximity-based game of tag using lumalive e-textile displays. In CHI'11 Extended Abstracts on Human Factors in Computing Systems. ACM, 1147-1152.

[12] Adrian David Cheok, Kok Hwee Goh, Wei Liu, Farzam Farbiz, Siew Wan Fong, Sze Lee Teo, Yu Li, and Xubo Yang. 2004. Human Pacman: a mobile, wide-area entertainment system based on physical, social, and ubiquitous computing. Personal and ubiquitous computing 8, 2 (2004), 71-81.

[13] Nigel Cross. 2006. Designerly ways of knowing. Springer.

[14] Euan Freeman, Graham Wilson, Stephen Brewster, Gabriel Baud-Bovy, Charlotte Magnusson, and Hector Caltenco. 2017. Audible Beacons and Wearables in Schools: Helping Young Visually Impaired Children Play and Move Independently. In Proceedings of the 2017 CHI Conference on Human Factors in Computing Systems. ACM, 4146-4157.

[15] Bill Gaver and John Bowers. 2012. Annotated portfolios. interactions 19, 4 (2012), $40-49$.

[16] Francine Gemperle, Chris Kasabach, John Stivoric, Malcolm Bauer, and Richard Martin. 1998. Design for wearability. In Wearable Computers, 1998. Digest of Papers. Second International Symposium on. IEEE, 116-122.

[17] Ç Genç, OT Buruk, Sİ Yılmaz, K Can, and O Özcan. 2018. Exploring computational materials for fashion: Recommendations for designing fashionable wearables. International fournal of Design 12, 3 (2018), 1-19.

[18] Rúben Gouveia, Fábio Pereira, Evangelos Karapanos, Sean A Munson, and Marc Hassenzahl. 2016. Exploring the design space of glanceable feedback for physical activity trackers. In Proceedings of the 2016 ACM International foint Conference on Pervasive and Ubiquitous Computing. ACM, 144-155.

[19] Will Greenwald. 2018. Nintendo Labo Robot Kit. https://uk.pcmag.com/ nintendo-labo-toy-con-02-robot-kit-for-nintendo-switch/93175/review/ nintendo-labo-robot-kit

[20] Robin Hunicke, Marc LeBlanc, and Robert Zubek. 2004. MDA: A formal approach to game design and game research. In Proceedings of the AAAI Workshop on Challenges in Game AI, Vol. 4. 1722

[21] HaptX Inc. 2018. HaptX VR Glove. https://haptx.com

[22] Katherine Isbister, Kaho Abe, and Michael Karlesky. 2017. Interdependent Wearables (for Play): A Strong concept for design. In Proceedings of the $2017 \mathrm{CHI}$ Conference on Human Factors in Computing Systems. ACM, 465-471.

[23] Katherine Isbister, Elena Márquez Segura, and Edward F Melcer. 2018. Social Affordances at Play: Game Design Toward Socio-Technical Innovation. In Proceedings of the 2018 CHI Conference on Human Factors in Computing Systems. ACM, 372.

[24] Ke Jing, Natalie Nygaard, and Theresa Jean Tanenbaum. 2017. Magia Transformo: Designing for Mixed Reality Transformative Play. In Extended Abstracts Publication of the Annual Symposium on Computer-Human Interaction in Play. ACM, 421-429.

[25] Keith Johnstone. 2012. Impro: Improvisation and the theatre. Routledge.

[26] Viirj Kan, Katsuya Fujii, Judith Amores, Chang Long Zhu Jin, Pattie Maes, and Hiroshi Ishii. 2015. Social textiles: Social affordances and icebreaking interactions through wearable social messaging. In Proceedings of the Ninth International Conference on Tangible, Embedded, and Embodied Interaction. ACM, 619-624.

[27] Markus Klann. 2007. Playing with fire: Participatory design of wearable computing for fire fighters. In CHI'07 Extended Abstracts on Human Factors in Computing Systems. ACM, 1665-1668.
[28] Heli Koskimäki, Pekka Siirtola, Eino Keskitalo, Lauri Tuovinen, Tim Luoto, Riikka Ahola, Riitta Pyky, Raija Korpelainen, Timo Jämsä, Hannu Heikkinen, et al. 2017. Computer game and wearable sensors based approach to promote physical activity for young men. In Proceedings of the 2017 ACM International Foint Conference on Pervasive and Ubiquitous Computing and Proceedings of the 2017 ACM International Symposium on Wearable Computers. ACM, 512-517.

[29] Sven Kratz and Michael Rohs. 2009. HoverFlow: expanding the design space of around-device interaction. In Proceedings of the 11th International Conference on Human-Computer Interaction with Mobile Devices and Services. ACM, 4.

[30] Virtual Motion Labs. 2018. Virtual Motion Labs. http://www.virtualmotionlabs. com

[31] Paul Lamkin. 2016. Pokémon Go Plus Wearable Sells Out: Now Selling For Silly Money On Ebay. https://www.forbes.com/sites/paullamkin/2016/07/11/ pokemon-go-plus-wearable-sells-out-now-selling-for-silly-money-on-ebay/ $\{\#\} 9 \mathrm{~b} 1751 \mathrm{f} 20 \mathrm{~b} 43$

[32] Sam Loveridge. 2015. Why the Fallout 4 Pip Boy Edition is a huge disappointment. http://www.trustedreviews.com/opinion/ why-the-fallout-4-pip-boy-edition-is-a-huge-disappointment- 2926374

[33] Jonas Löwgren. 2013. Annotated portfolios and other forms of intermediate-level knowledge. Interactions 20, 1 (2013), 30-34.

[34] Elena Márquez Segura, James Fey, Ella Dagan, Samvid Niravbhai Jhaveri, Jared Pettitt, Miguel Flores, and Katherine Isbister. 2018. Designing Future Social Wearables with Live Action Role Play (Larp) Designers. In Proceedings of the 2018 CHI Conference on Human Factors in Computing Systems. ACM, 462.

[35] Thomas Martin, Mark Jones, Josh Edmison, and Ravi Shenoy. 2003. Towards a design framework for wearable electronic textiles. In null. IEEE, 190.

[36] Tiago Martins, Teresa Romão, Christa Sommerer, Laurent Mignonneau, Nuno Correia, and Quinta Torre. 2008. Towards an Interface for Untethered Ubiquitous Gaming. In Proceedings of the 2008 Advances in Computer Entertainment Technology - ACE' 08. 26-33.

[37] Jin Moen. 2007. From hand-held to body-worn: embodied experiences of the design and use of a wearable movement-based interaction concept. In Proceedings of the 1st international conference on Tangible and embedded interaction. ACM, 251-258.

[38] Florian'Floyd' Mueller, Richard Byrne, Josh Andres, and Rakesh Patibanda. 2018. Experiencing the Body as Play. In Proceedings of the 2018 CHI Conference on Human Factors in Computing Systems. ACM, 210

[39] Florian'Floyd' Mueller, Martin R Gibbs, Frank Vetere, and Darren Edge. 2017. Designing for bodily interplay in social exertion games. ACM Transactions on Computer-Human Interaction (TOCHI) 24, 3 (2017), 24.

[40] Jörg Müller, Florian Alt, Daniel Michelis, and Albrecht Schmidt. 2010. Requirements and design space for interactive public displays. In Proceedings of the 18th ACM international conference on Multimedia. ACM, 1285-1294.

[41] Nintendo. 2018. Nintendo Labo Robot Kit. https:/labo.nintendo.com/kits/ robot-kit/

[42] Eleuda Nunez, Francesco Visentin, and Kenji Suzuki. 2016. Friend ${ }^{*}$ Chip: A Bracelet with Digital Pet for Socially Inclusive Games for Children. In Proceedings of the 29th Annual Symposium on User Interface Software and Technology. ACM, 213-214.

[43] Stuart Reeves, Steve Benford, Claire O'Malley, and Mike Fraser. 2005. Designing the spectator experience. In Proceedings of the SIGCHI conference on Human factors in computing systems. ACM, 741-750.

[44] Kay Romer and Friedemann Mattern. 2004. The design space of wireless sensor networks. IEEE wireless communications 11, 6 (2004), 54-61.

[45] Katie Salen, Katie Salen Tekinbaş, and Eric Zimmerman. 2004. Rules of play: Game design fundamentals. MIT press.

[46] Jesse Schell. 2014. The Art of Game Design: A book of lenses. AK Peters/CRC Press.

47] Donald A Schön. 2017. The reflective practitioner: How professionals think in action. Routledge.

[48] Hoyeong Song, Soobin Lee, Hyunsoo Kim, Gunmin Jang, Younggeun Choi, and Dongseok Yang. 2016. Rapael: Wearable technology and serious game for rehabilitation. In Proceedings of the 2016 CHI Conference Extended Abstracts on Human Factors in Computing Systems. ACM, 3774-3777.

[49] Theresa Jean Tanenbaum and Jim Bizzocchi. 2009. Rock Band: a case study in the design of embodied interface experience. In Proceedings of the 2009 ACM SIGGRAPH Symposium on Video Games. ACM, 127-134.

50] Theresa Jean Tanenbaum and Karen Tanenbaum. 2015. Envisioning the Future of Wearable Play: Conceptual Models for Props and Costumes as Game Controllers.. In FDG.

[51] Oscar Tomico, Lars Hallnäs, Rung-Huei Liang, and Stephan AG Wensveen. 2017. Towards a next wave of wearable and fashionable interactions. International Journal of Design 11, 3 (2017), 1-6.

[52] Loïs Vanhée, Elena Márquez Segura, and Katherine Isbister. 2018. Firefly: A Social Wearable to Support Physical Connection of Larpers. In Extended Abstracts of the 2018 CHI Conference on Human Factors in Computing Systems. ACM, D311.

[53] Annika Waern, Markus Montola, and Jaakko Stenros. 2009. The three-sixty illusion: designing for immersion in pervasive games. In Proceedings of the SIGCHI Conference on Human Factors in Computing Systems. ACM, 1549-1558.

[54] Clint Zeagler. 2017. Where to wear it: functional, technical, and social considerations in on-body location for wearable technology 20 years of designing for 
wearability. In Proceedings of the 2017 ACM International Symposium on Wearable Computers. ACM, 150-157.

[55] Clint Zeagler. 2019. Where to Wear It : Wearable Technology BODY MAPS http://www.clintzeagler.com/where-it-body-maps/

[56] John Zimmerman, Jodi Forlizzi, and Shelley Evenson. 2007. Research through design as a method for interaction design research in HCI. In Proceedings of the SIGCHI conference on Human factors in computing systems. ACM, 493-502. 